\title{
Cornisa: conductas suicidas en los adolescentes del décimo año de educación básica
}

\author{
Cornish: suicide behaviors in the teens of the tenth grade of basic education \\ Cornisa: Condutas suicidas nos adolescentes do décimo ano de Educação Básica
}

DOI: https://doi.org/10.21803/pensam.v12i22.252

Milton Patricio Guallpa Guallpa

https://orcid.org/0000-0002-8785-2999

Sergio Constantino Ochoa Encalada

https://orcid.org/0000-0003-3067-3719

\section{¿Cómo citar este artículo?}

Guallpa, M. \& Ochoa, S. (2019). Cornisa: conductas suicidas en los adolescentes del décimo año de educación básica. Pensamiento Americano, 12(23) 145-153.

DOI: https://doi.org/10.21803/pensam.v12i22.252

\section{Resumen}

Los índices de suicidio y de ideación suicida en los adolescentes es un problema que ha sufrido una adición considerable durante los últimos años, debido a que cada vez es más común que los adolescentes tomen la decisión de atentar contra su vida como una forma de escape, ante los problemas que están pasando, la tasa de intentos suicidas es un problema que a queja a las sociedades desde tiempo inmemoriales, ya que este es visto por quienes optan por este mecanismo, como medio de escape a la realidad a la cual se enfrentan. El presente trabajo determina las posibles conductas suicidas en los discentes del décimo año de educación básica de la Unidad Educativa "Luis Cordero", para poder conocer si hay índices de ideación suicida, se utilizó el inventario de orientaciones suicidas ISO 30, adaptación realizada por la Dra. María Martina Casullo y su equipo en la Universidad de Buenos Aires en 1998, de la descripción de Orientaciones Suicidas de King \& Kowalchuk (1994- USA). Se aplicó 107 inventarios, de los cuales el $51 \%$ que corresponde a 64 estudiantes, se identifica que tienen un alto índice de ideación suicida.

Palabras Clave: Suicidio, ideación suicida, familia, aceptación social, adolescencia

\begin{abstract}
The rates of suicide and suicidal ideation in adolescents is a problem that has suffered a considerable addition in recent years, because it is increasingly common for adolescents to make the decision to attempt their life as a way of escape, given the problems that are happening, the rate of suicide attempts is an issue that has been complaining to societies since time immemorial, since this is seen by those who opt for this mechanism, as a means of escape from the reality they face. The present work determines the possible suicidal behaviours in the students of the tenth year of secondary education of the Educational Unit "Luis Cordero", to know if there are indexes of suicidal ideation, the inventory of suicidal orientations ISO 30 was used, adaptation carried out by the Dr María Martina Casullo and her team at the University of Buenos Aires in 1998, of the description of Suicide Orientations of King \& Kowalchuk (1994- USA). One hundred seven inventories were applied, of which 51\% corresponding to 64 students, are identified as having a high rate of suicidal ideation.
\end{abstract}

Key words: Suicide, suicidal ideation, family, social acceptance, adolescence.

*Artículo de reflexión 


\section{Resumo}

Os índices de suicídio e de ideação suicida nos adolescentes é um problema que cresceu consideravelmente durante os últimos anos devido a que é cada vez mais comum que os adolescentes tomem a decisão de atentar contra a sua própria vida como uma forma de escape frente aos problemas que estão passando. A taxa de tentativas de suicídios é um problema que afeta a sociedade desde tempos imemoriais. $O$ presente trabalho determina as possíveis condutas suicidas nos discentes do décimo ano de educação básica da Unidade Educativa "Luis Cordero"; para poder conhecer se existem índices de ideação suicida, se utilizou um inventário de orientações suicidas ISSO 30, uma adaptação feita pela Dra. Maria Martina Casullo e sua equipe na Universidade de Buenos Aires em 1998, a partir da descrição de Orientações Suicidas de King \& Kowalchuk (1994-USA). Foram aplicados 107 inventários, dos quais 51\% correspondem a 64 estudantes, e se identifica que possuem um alto índice de ideação suicida.

Palavras-chave: Suicídio, ideação suicida, família, aceitação social, adolescência.

\section{Perfil}

$146]$ Universidad de Cuenca sede Azogues. mitonmetal@gmail.com.

Milton Patricio Guallpa

Guallpa

Licenciado en Ciencias de la

Educación

\section{Perfil}

Universidad de Cuenca sede Azogues. scochoae@ucacue.edu.ec

Sergio Ochoa Encalada

Magister en Psicopedagogía

Talentos y Creatividad 


\section{Introducción}

"La conducta suicida puede ser definida como un acto de preocupación, un acto de provocarse así mismo un daño, la finalidad que tiene esta es de inducir la muerte, de causarse un gran daño físico. La conducta suicida es un acto comprendido desde la ideación de un suicidio que puede ser consumido, hasta la decisión de proceder a ejecutar la conducta, es por esto que el suicidio es una de las principales causas de mortalidad en los adolescentes. Por otro lado, las conductas suicidas no letales es un posible factor de riesgo para un futuro suicidio, estas son señales directas de que el adolescente esta pasado por una situación tensa en la cual las auto lesiones son una vía de escape y desahogo" (Silva, Vicente, Saldivia \& Kohn, 2013).

La ideación suicida es un acto premeditado y planificado, por el individuo que va a suicidarse, muchas veces las personas del entorno no logran identificar los factores que llegan a ser señales que se debe considerar de importancia para evitar esos actos, aquí juega un papel preponderante el tiempo, por que la persona con esta ideación pasa por diferentes etapas que van de manera progresiva. Existen ciertas conductas que se debe tomar en cuenta como comportamientos de aislamiento, autolesiones, cambios drásticos en la rutina buscando la soledad y un marcado encierro social.

Los factores que desencadenan un comportamiento suicida se agravan de debido a que los padres de familia del estudiante le dan una mínima atención a este problema llegando a creer que esto debe únicamente a comportamientos ligados a una adolescencia conflictiva, es por eso que la ideación suicida debido a su grado de seriedad y peligrosidad muy marcada, por lo tanto, los padres deben ser los primeros en actuar ante un posible indicio.
"Las amenazas de un posible suicida son sus gestos e ideación sobre la vida, por lo general no son tomados en cuenta por las figuras paternas dentro del núcleo familiar del joven, siendo una forma de hacer llegar un mensaje de dolor angustia y sufrimiento a través de actos autodestructivos (Vargas \& Saavedra, 2012).

La Unidad Educativa Luis Cordero, por ser un centro de estudios, es el lugar donde la mayor parte de tiempo pasan los adolescentes, siendo indispensable que los docentes conozcan las características de esta problemática, para evitar en un futuro que la ideación se convierta en un acto consumado; todo depende con que antelación se detectó el problema, y buscar posibles soluciones. No existe trabajos parecidos realizados en el centro educativo y a nivel provincial, una investigación realizada en Parú, tiene similitud sobre la ideación suicida y cohesión familiar en estudiantes preuniversitarios entre 15 y 24 años Lima 2005, La presente tiene como objetivo determinar la ideación suicida y su asociación con el pertenecer a familias de muy bajos de cohesión familiar en estudiantes entre 15 a 24 años (Muñoz, Pinto, Callata, Napa \& Perales, 2006).

La población investigada corresponde a adolescentes la Unidad Educativa Luis Cordero del décimo año de educación básica, que corresponde a la edad de 14 a 15 años, por considerarse una etapa detracción y cambios continuos y llenos de desafíos, no necesariamente negativas e inciertos, al menos que los contextos socio cultural o neurobiológicas específicos que lo provoquen, las prácticas de transición alteran el equilibrio emocional y la percepción personal y objetiva de bienestar (Gómez \& Cogollo, 2010).

Debido a la gran importancia que tiene el tema, se abordará la incidencia directa que tienen estas conductas frente a los hogares 
disfuncionales, la aceptación social, como factores de riesgos.

\section{Metodología}

Los métodos utilizados para la presente investigación fue el descriptivo, que sirvió como sustento teórico, en relación a la identificación de conductas suicidas o ideación suicida en los estudiantes del décimo año de educación básica de la unidad educativa Luis Cordero de la ciudad de Azogues, y el método cuanti-cualitativo para la tabulación de resultados.

Se aplicó a una población de 107 adolescentes que cursan el décimo año de educación general básica durante el periodo lectivo 2016-2017. Para la recopilación de antecedentes se manejó el inventario ISO 30 que es una adaptación del inventario de Orientaciones Suicidas de King \& Kowalchuk (1994- USA) realizada en la Universidad de Buenos Aires en 1998 por la Dra. María Martina Casullo y equipo (Bodon \& Rios, 2016).

El inventario consta de 30 ítems que deben ser respondidos con una escala de cuatro opciones. Las opciones se evalúan de 0 a 3 puntos. La escala permite evaluar los niveles de cinco factores asociados al riesgo suicida: Desesperanza, baja autoestima, incapacidad para afrontar emociones, aislamiento social, ideación suicida. Para la obtención de resultados se tomó en cuenta un puntaje global que va del análisis del puntaje bruto con especial consideración de los ítems críticos permite llegar a la clasificación del riesgo suicida en tres niveles: Bajo (O a 30), moderado (= ó > 30) y alto (= ó > 45).

Se analizaron y tabularon las respuestas del inventario ISO 30, mismas que fueron las siguientes:
Tabla 1

Ideación Suicida

\begin{tabular}{l|ll}
\hline Indicadores & Frecuencia & Porcentaje \\
\hline Bajo & 5 & $5 \%$ \\
Moderado & 43 & $40 \%$ \\
Alto & 60 & $56 \%$ \\
\hline
\end{tabular}

Fuente: Investigación de campo (Inventario aplicado a los estudiantes del décimo año de educación básica de la Institución educativa Luis Cordero

Elaborado por: Milton Gaullpa; Mgs Sergio Ochoa

En el presente cuadro se observa que el $56 \%$ de encuestados tienen un rango alto de ideación suicida, un $40 \%$ se encuentran dentro de un rango medio y un porcentaje de 5\% dentro de un rango bajo referente a la ideación suicida.

Tabla 2.

Probabilidades de ideación

\begin{tabular}{l|ll}
\hline INDICADORES & FRECUENCIA & PORCENTAJE \\
\hline 0. Totalmente en desacuerdo & 11 & $10 \%$ \\
1. en parte en desacuerdo & 14 & $13 \%$ \\
2. en parte de acuerdo & 32 & $30 \%$ \\
3. totalmente de acuerdo & 51 & $47 \%$ \\
\hline
\end{tabular}

Fuente: Investigación de campo (Inventario aplicado a los estudiantes del décimo año de educación básica de la Institución educativa Luis Cordero

Elaborado por: Milton Guallpa; Mgs Sergio Ochoa

Sobre la pregunta: hay muchas posibilidades para mí de ser feliz en el futuro, los 51 estudiantes que corresponde al porcentaje de $47 \%$ están totalmente de acuerdo con el hecho de que tienen muchas posibilidades para ellos de ser felices, con un porcentaje de $30 \%$ que son 32 estudiantes, nos dice que están en parte de acuerdo con el hecho antes mencionado, un 13\% que es igual 14 estudiantes dicen estar en parte en desacuerdo, mientras que un 10\% que equivale 11 estudiantes dicen estar completamente en desacuerdo. 
Tabla 3.

Sentimientos de desesperanza

\begin{tabular}{l|ll}
\hline INDICADORES & FRECUENCIA & PORCENTAJE \\
\hline 0. Totalmente en desacuerdo & 12 & $11 \%$ \\
1. en parte en desacuerdo & 28 & $26 \%$ \\
2. en parte de acuerdo & 38 & $35 \%$ \\
3. totalmente de acuerdo & 30 & $28 \%$ \\
\hline
\end{tabular}

Fuente: : Investigación de campo (Inventario aplicado a los estudiantes del décimo año de educación básica de la Institución educativa Luis Cordero. Elaborado por: Milton Guallpa; Mgs Sergio Ochoa.

Con respecto a, cuando me pasa algo malo siento que mis sentimientos, esperanzas de una vida mejor son poco reales, con un porcentaje de $35 \%$ que equivale a 38 estudiantes dicen se encontrarse en parte de acuerdo, un porcentaje del 38\% que es igual a 30 encuestados nos dice que se encuentran en parte de acuerdo, el $26 \%$ de ellos que equivale a 30 estudiantes no dice estar en parte de acuerdo, mientras que se evidencia que un porcentaje del $11 \%$ que equivale a 12 estudiantes dicen estar totalmente de acuerdo con el enunciado.

Tabla 4.

Suicidio como una solución de escape

\begin{tabular}{l|ll}
\hline INDICADORES & FRECUENCIA & PORCENTAJE \\
\hline 0. Totalmente en desacuerdo & 58 & $54 \%$ \\
1. en parte en desacuerdo & 22 & $20 \%$ \\
2. en parte de acuerdo & 18 & $17 \%$ \\
3. totalmente de acuerdo & 10 & $9 \%$ \\
\hline
\end{tabular}

Fuente: : Investigación de campo (Inventario aplicado a los estudiantes del décimo año de educación básica de la Institución educativa Luis Cordero.

Elaborado por: Milton Guallpa; Mgs Sergio Ochoa

En lo referente, para impedir que las cosas empeoren creo que suicidarse es la solución, se evidencia que con un $54 \%$ que equivale a 58 sujetos, están totalmente en desacuerdo, un porcentaje de $20 \%$ igual a 22 estudiantes dicen que estar en parte de acuerdo, $17 \%$ de los encuestados que serían unos 18 de ellos menciona estar en parte de acuerdo, mientras que el 9\% de ellos está completamente de acuerdo con el cuestionamiento.

\section{Discusión}

El suicidio debe ser considerado un hecho de causa multifactorial en el que intervienen factores biológicos, psicológicos y sociales, este último es una expresión de una falla de mecanismos adaptativos del sujeto en su medio ambiente, provocada por la situación conflictiva actual o permanente que genera un estado de tensión emocional. Es así que en la investigación existe un porcentaje de $56 \%$ de encuestados se encuentran dentro de un rango alto de ideación suicida, las causas que desencadenan una ideación suicidad son variadas y de desglosan en otras conductas autodestructivas en el estudiante, como posibles cuadros de depresión o haciendo que el sujeto se aislé de su medio social (Arlaes Nápoles, Hernández, Álvarez \& Cañizares, 1998).

El comportamiento suicida es una de las principales causas de muerte en el mundo, estadísticamente cada día se suicidan más de mil personas y lo intentan hacerlo cientos de miles. El comportamiento suicida es un serio problema de salud mental y una problemática social, que va teniendo un considerable aumento con el trascurso de las generaciones. A sabiendas que el problema de la ideación suicida es una complicación grave ya que sus consecuencias son directas, el establecimiento debe de generar programas y talleres en donde se traten temas relacionados con la autoestima y auto superación ya que son puntos fundamentales para el diagnóstico.

Con respecto a la pregunta ¿Cuándo me pasa algo malo siento que mis expectativas de vida son poco reales?, dentro de la institución educativa el $28 \%$ de los adolescentes están totalmente de acuerdo, a pesar de ser un porcentaje bajo, el resultado es preocupante, porque se denota la inseguridad, que sus metas no se llegan a cumplir, que no sean aceptados dentro de un grupo, etc. 
La funcionalidad de la familia juega un papel preponderante dentro de las expectativas de vida que tiene un adolescente, mejorándolas, o por lo contrario haciéndola desesperanzadora sin un futuro claro, la función que cumple esta es procurar que dentro de su núcleo no existan conflictos que puedan alterar el buen funcionamiento emocional de cada uno de ellos. Como resultado emanan familias disfuncionales, cuyos miembros no solo viven un traslucimiento de valores, sino tienen dificultad de afrontar la autocrítica.

Algunas investigaciones sobre la ideación suicida reflejan que esta tiene una íntima relación con características psicológicas, demográficas y sociales, sobre los factores de orden cultural y étnico, estos estudios expresan la diferencia entre la ideación suicida de estudiantes de zonas rurales y urbanas. En base a los resultados obtenidos con un 56\% de ideación suicidad en la institución educativa que se encuentra en la ciudad.

En cuanto a las características socioculturales uno de los mayores factores que inciden en el índice de ideación suicida, son los niveles bajos de instrucción educativa, que viven en zonas marginadas con una situación económica deficiente. Dentro de esta característica también se engloba las condiciones sociales e interpersonales adversas como el acoso, la intimidación y el bullyng. Si hablamos dentro de las características psicológicas que conlleva el suicidio se puede mencionar la existencia de antecedentes psicopatológicos previos y trastornos mentales de importancia. Este último no puede ser un causante directo de la ideación suicida ya que el suicidio es un acto multifactorial y no se le pude hacer una conclusión premeditada con solo un factor que pueda asociarse con el suicidio.

Estas organizaciones familiares son endebles, los provoca efectos graves en los años de ado- lescencia de un joven. Si se fragmenta la familia al menos provoca una precariedad de los vínculos intrafamiliares (Hernández-Castillo, Cargill-Foster \& Gutiérrez-Hernández, 2011).

Krauskopof (1999), sostiene que el adolescente se traza la enunciación personal y social del ser humano a través de una segunda determinación que moviliza procesos, de tal afirmación se hace pernicioso, pues los y las adolescentes son portadores de cambios culturales. Exploración, diferenciación del entorno familiar, búsqueda de pertenencia y sentido de su vida. Demandando para el medio adulto, una reacomodación de esquemas arcaicos psicosociales que incluyen el manejo de nuevos modelos de autoridad innovadores para llevar sus vidas.

Un porcentaje de 35\% que equivale a 38 de los estudiantes concuerdan estar en parte de acuerdo sobre, cuando me pasa algo malo siento que mis esperanzas de una vida mejor son poco reales, dándonos a conocer que los adolescentes tienden a tener pocas esperanzas alejándose a como ellos lo tenían planeado. En lo que respecta a la ideación de una vida mejor se pude constatar que los sujetos se desmoronan cuando sus vidas van cuesta abajo por problemas relacionados a cuestiones, familiares, educativas, o sociales.

Para impedir que las cosas empeoren, referente a la pegunta creo que suicidarse es la solución, un porcentaje del 9\% que equivale a 10 estudiantes encuestados, se constata que están totalmente de acuerdo para que las cosas emporen una solución factible es la de optar por el suicidio. Estos resultados dan a conocer que la mayoría de la población de la cual se utilizó para recolectar datos opina sobre el suicidio como una posible solución para terminar con sus problemas. Cortés Alfaro (2014), Menciona que dentro de la adolescencia se aumentan las cargas de presiones 
y responsabilidades individuales, esto combinado con la inexperiencia que todavía poseen, siendo vulnerables a cometer tropiezos y malas decisiones, pasando por momentos de angustia, soledad y frustración llevando a cometer un acto o conducta suicida.

En cuanto a la expectativa de vida que tiene los estudiantes, se puede decir que en la mayoría de ellos se encuentran con pocas posibilidades que sus perspectivas mejoren para bien, dado que tienen una visión muy desesperanzadora de su posibilidad para poder tener una vida placentera, esto constituye uno de los motivos para que los jóvenes tomen la decisión de acabar con sus vidas por medio de un suicidio.

Los resultados evidenciados en lo que respecta a cuando mi vida no transcurre fácilmente estoy dominado por una confusión de sentimientos, se pudo evidenciar que un 34\% de encuestados, están en parte de acuerdo con el cuestionamiento antes mencionado. Álvarez Zúñiga, Ramírez Jacobo, Silva Rodríguez, Coffin Cabrera \& Jiménez (2009) Sostienen que a sabiendas de que la adolescencia es una etapa netamente conflictiva, un periodo lleno de modificaciones a nivel biológico, epistémico y sindical.

Los resultados obtenidos en la mayoría de adolescentes han considerado al suicidio como un acto de salida, un medio para poder terminar con los problemas que les aqueja, hecho que a lo largo de la historia fue considerada únicamente una práctica concebida por mujeres, pero esto fue un mito descartado ya que si no es en un porcentaje similar en hombre hay una marcada incidencia por parte de ellos para cometer un acto suicida.

Vasquez (2010), menciona que en la etapa de la adolescencia se presenta un carácter muy impulsivo lo cual hace que en muchas de las ocasiones estas propicien un comportamiento inadecuado, en la mayoría de las veces, el joven trata de terminar con su vida, lo que en realidad puede significar es que este está tratando de evadir la realidad por la cual está pasando. Como prueba de esto se evidencia dentro del cuadro de estadísticas general existe un gran porcentaje de estudiantes que tienen un alto grado de ideación suicida. En el resultado de la calificación del test se obtuvo que con un porcentaje de 50\% de encuestados, tienen un alto indicio de ideación suicida. La mayor incidencia del intento suicida se obtuvo en los estudiantes de entre 14 a 16 años, Vasquez (2010), sostiene que es frecuente que dentro de estas edades existen tendencias por parte de los jóvenes a cometer actos en los cuales se auto infrinjan daño, debiéndose a la mal llamada crisis de la juventud, está bien acompañada de conflictos y trastornos emocionales.

Duarte Vélez, Lorenzo-Luaces y Rosselló (2012) aluden que los posibles pensamientos suicidas que se pueden hacer presentes en los estudiantes son un factor de gran riesgo, entre estos pueden ser ideaciones de hacerse lesiones físicas y cortaduras, con objetos corto punzantes, haciéndose presente en un adolescente se ve inmiscuido en problemas que pueden generarle un alto grado de estrés, donde en joven se ve atado de manos y no puede hacer nada al respecto. La institución educativa debe tomar cartas en el asunto, los estudiantes del décimo año de educación básica donde se presentan elevados rangos de ideación suicida.

\section{Conclusiones}

- La ideación suicida dentro de la institución educativa está presente con un porcentaje de $56 \%$ de los estudiantes. la institución educativa debe dar mayor a esta problemática. 
- Una de las causas que originan comportamientos suicidas en los adolescentes es una disfuncionalidad dentro del núcleo familiar, haciendo énfasis en agresiones físicas verbales.

- Las instituciones educativas deben formular programas en donde conjuntamente con los padres de familia personal docente trabajen conjuntamente en dar mayor atención a este sector.

\section{Referencias}

Álvarez Zúñiga, M., Ramírez Jacobo, B., Silva Rodríguez, A., Coffin Cabrera, N. \& Jiménez Rentería, M. (2009). La relación entre depresión y conflictos familiares en adolescentes. International Journal of Psychology and Psychological Therapy, 9 (2), 205-216.

Arlaes Nápoles, L., Hernández, G., Álvarez, D. \& Cañizares, T. (1998). Conducta suicida: factores de riesgo asociados. Revista Cubana de Medicina General Integral, $14(2), 122-126$.

Bodon, M. C. \& Ríos, M. A. (2016). Lineamientos generales para comprensión, detección y prevención. Lima, Perú. Recuperado de: http://www.psi.uba.ar/academica/carrerasdegrado/psicologia/sitios_catedras/ obligatorias/070_psicoterapias1/material/suicidio_lineamientos.pdf

Cortés Alfaro, A. (2014). Conducta suicida adolescencia y riesgo. Revista Cubana de Medicina General Integral, 30(1), 132-139.

Duarte Vélez, Y., Lorenzo-Luaces, L. \& Rosselló, J. (2012). Ideación suicida: Síntomas depresivos, pensamientos disfuncionales, auto- concepto, y estrategias de manejo en adolescentes puertorriqueños/as. Revista Puertorriqueña de Psicología, 23, 1-17.

Gómez, E. \& Cogollo, Z. (2010). Factores predictores relacionados con el bienestar general en adolescentes. Salud Pública. 12(1). 61-70.

Hernández-Castillo, L., Cargill-Foster, N., \& Gutiérrez-Hernández, G. (2012). Funcionalidad familiar y conducta de riesgo en estudiantes de nivel medio superior Jonuta, Tabasco 2011. Salud en Tabasco, 18 (1), 14-24.

Krauskopof, D. (1999). El desarrollo psicológico en la adolescencia: las transformaciones en una época de cambios. Adolescencia y Salud, 1(2), 23-31.

Muñoz M. J., Pinto M., Callata C., Napa D. \& Perales C. (2006). Ideación suicida y cohesión familiar en estudiantes preuniversitarios entre 15 y 24 años, Lima 2005. Revista Peruana de Medicina Experimental y Salud Pública, 23 (4), 239-246.

Silva, D., Vicente, B., Saldivia, S. \& Kohn, R. (2013). Conduc- 
ta suicida y trastornos psiquiátricos en Chile, un estudio poblacional. Revista médica de Chile, 141(10), $1275-1282$.

Vargas, H. \& Saavedra, J. (2012). Factores asociados con la conducta suicida en adolescentes. Revista de Neuro-Psiquiatría, 75 (1), 19-28.

Vasquez, M. J. (2010). Factores de riesgo suicida en adolescentes. Obtenido de Scielo: http://www.scielo.org/ php/index.php?lang=es 